\title{
Direito à Saúde e Cobertura universal de saúde
}

\section{Matheus Zuliane Falcão}

Dissertação apresentada à Banca Examinadora do Programa de Pós-graduação da Faculdade de Direito da Universidade de São Paulo para obtenção do título de Mestre em Direito.

Área de concentração: Direitos Humanos Orientadora: Prof. Dr. Fernando Mussa Abujamra Aith

São Paulo, 2020 
Catalogação da Publicação

Serviço de Biblioteca e Documentação

Faculdade de Direito da Universidade de São Paulo

Falcão, Matheus zuliane

Direito à Saúde e Cobertura universal de saúde Matheus Zuliane Falcão ; orientador Fernando Mussa Abujamra Aith -- São Paulo, 2020.

181

Dissertação (Mestrado - Programa de Pós-Graduação em Direitos Humanos) - Faculdade de Direito, Universidade de São Paulo, 2020.

1. Direito à Saúde. 2. Saúde Global. 3. Cobertura universal. 4. Direito Sanitário. I. Aith, Fernando Mussa Abujamra, orient. II. Título. 
Nome: Matheus Zuliane Falcão

Título: Direito à Saúde e Cobertura universal de saúde

Dissertação apresentada à Faculdade de Direito da Universidade de São Paulo como exigência parcial para obtenção do título de Mestre em Direito.

Aprovado em:

Banca examinadora

Prof. Dr. Instituição:

Julgamento: Assinatura:

Prof. Dr. Instituição:

Julgamento: Assinatura:

Prof. Dr. Instituição:

Julgamento: Assinatura: 
À minha mãe, Silene.

A meu pai, Paulo.

Uma singela homenagem em agradecimento eterno a todos os esforços que empreenderam pela minha educação. 


\section{Agradecimentos}

Agradecer será sempre um ato incompleto. As pessoas e as situações que até aqui me trouxeram tendem ao infinito. Desta trilha, desconfio desconhecer grande parte. Do que não ignoro, se fosse listar tudo, faria uma outra dissertação. Começo, portanto, reconhecendo que este trabalho, mais que ponto de chegada, é um caminho percorrido e que sou grato por cada passo. Dedico este sentimento maior de gratidão à força divina, que permitiu e inspirou meu corpo, minha mente e minha alma a estarem onde estiveram.

Memoro, na sequência, algumas pessoas, que por meio de seus atos, contribuíram diretamente com este trabalho.

Agradeço à minha mãe, Silene, ao meu pai, Paulo, por toda parceria em meu trajeto educacional e todo suporte desde minha primeira infância. $\mathrm{O}$ valor que a educação teve em minha casa já é mais do que suficiente para que dedique este trabalho integralmente a vocês. Aqui agradeço também à Paula, minha irmã, pelos segredos que compartilhamos.

Agradeço aos mestres acadêmicos, com a certeza de que muitas linhas deste trabalho têm mais deles do que de mim e de que cada qual concedeu-me uma peça deste quebra-cabeça inconcluso que tento montar no meu percurso acadêmico.

Primeiramente ao meu orientador, Prof. Fernando Aith, pela inspiração, orientação, parceria, generosidade, pelas oportunidades e pela imensa compreensão com as minhas falhas e meus percursos intensos ao longo dos anos de mestrado

À Profa. Sueli Dallari, por ser minha maior referência da articulação teórica entre direito, saúde e direito à saúde e por toda generosidade comigo desde meus primeiros passos no CEPEDISA.

À Prof. Deisy Ventura e ao Dr. Félix Rigoli, por serem inspirações acadêmicas e profissionais e por suas grandes contribuições em minha banca de qualificação.

Agradeço a cinco pessoas que foram decisivas para a formulação e a conclusão deste trabalho: À Ana Navarrete, por sua compreensão e generosidade decisivas nos primeiros e nos últimos passos. À Laís Vignati, por ter pintado o branco e preto do conceito de saúde com as cores do cuidado e por não me deixar esquecer jamais que as pessoas são o nosso ponto de partida e o nosso ponto de chegada. Ao Vitor Ido, por todo apoio e por ser meu principal interlocutor neste trabalho e em vários dos passos ao longo 
destes anos de mestrado. À Marlyna Nalbandian, por, mesmo a quilômetros de distância, ter me ouvido inúmeras vezes em horas de incerteza e por ter me ensinado o poder das maçãs no manejo da ansiedade. À Raissa, por ser a principal parceira na fase de escrita, dedicando madrugadas à consecução deste trabalho comigo e ter me salvado de me perder na ansiedade típica do mestrado.

Tão decisivo quanto na conclusão deste trabalho, agradeço à Roberta e ao Lucca, pela amizade expressa com a grande ajuda na revisão final.

Agradeço também à Clara, ao Iago e ao Hélio, por suas contribuições substanciais, em conversas, reflexões e conceitos, não apenas sobre saúde, mas sobre todo os aspectos que envolvem a execução de um mestrado.

Agradeço às parceiras acadêmicas e amigas querida, Angela Acosta, Marina Borba, Maria Eugênia e Lúcia Guerra, certo de que cada passo compartilhado em projetos e conversas estão refletidos no que deu certo nesta dissertação.

Agradeço aos parceiros de pós-graduação, pela partilha e pela ajuda que me concederam nesta reta final. Ao Marcelo, à Joana e novamente à Maria Eugênia.

Agradeço à Silmara Duarte, pela amizade neste trajeto e pelo apoio em vários momentos.

Dentre as experiências não acadêmicas que tive, certamente a que mais me ajudou a compreender meu objeto de pesquisa foi o ativismo junto ao Movimento pela Saúde dos Povos (People's Health Movement). Agradeço especialmente ao Gargeya, à Kriti, à Linda, à Ana Vracar, ao Emmanuel, ao Dan Owalla, ao Ravi Ram, ao Nafis Faizi, à Marta Giane, à Kátia Cesa, à Muna Odeh e ao Tinashe.

No mesmo sentido, agradeço a três parceiras do Centro de Brasileiro de Estudos da Saúde (CEBES), com quem partilhei diversas inquietações e que hoje tenho como queridas amigas Isabela, Jéssica e Alane. Agradeço ainda à Lígia Giovanella, por, no meio da $16^{\text {a }}$ Conferência Nacional de Saúde, ter muito gentilmente se disposto a conversar longamente comigo sobre o conceito de sistemas de saúde. 
"Reflecting again on your history and Constitution, the fullness of the right to health is still incomplete. Health not only encompasses the physical, mental and social wellbeing, but must be inclusive of spiritual well-being.

$$
[\ldots]
$$

Perhaps one day this notion of well-being can be included in the WHO definition of health?"

"When we review the right to health, we cannot help but notice that its global scope contains the hopes and aspirations of all the people of the world."

- Trechos do discurso do Reverendo Desmond Mpilo Tutu, Arcebispo Emérito da Cidade do Cabo, laureado com o Prêmio Nobel da Paz e grande liderança na luta contra o apartheid, na $61^{\mathrm{a}}$ reunião da Assembleia Mundial da Saúde. Genebra, 20 de maio de 2008 


\section{Resumo}

A Cobertura universal de saúde foi alçada a uma posição de alto prestígio no debate internacional sobre saúde no início do Século XXI, sendo indicada como o principal objetivo no setor. Ela se inicia da discussão sobre ampliação do acesso, financiamento e sistemas de saúde e nasce intimamente ligada aos conceitos de proteção financeira e de equidade, no entanto, passa a ser mencionada em diversos documentos internacionais, até se tornar o principal objetivo específico associado à saúde na Agenda de Desenvolvimento Sustentável das Nações Unidas (Agenda 2030), além de principal referência para vários outros documentos sobre política sanitária e sistemas de saúde, especialmente no âmbito de organizações internacionais com importante influência sobre políticas nacionais, como a OMS, seus escritórios regionais, o Banco Mundial e até organizações da sociedade civil. Este trabalho se propõe a articular o conceito de Cobertura universal de saúde no marco da efetivação do Direito à saúde, em outras palavras, buscamos entender em que medida, ela busca realizar algum dos aspectos do Direito à saúde e quais. Tomamos como hipótese que efetivação do Direito à saúde e cobertura universal não são sinônimos, mas que muitas vezes são tomados como tal ou como duas vias para o mesmo fim, que seria garantir saúde a todas e a todos, o que resultaria em duas possíveis distorções: restrição da efetivação do Direito à saúde a alguns de seus elementos ou promoção de uma resposta em saúde global para o fortalecimento dos sistemas de saúde que não condiz com o Direito à saúde. Para tal, após propor uma identificação dos elementos do Direito à saúde, realizamos análise documental das resoluções e relatórios das principais instâncias da OMS sobre o tema. Concluímos que a cobertura universal sofreu mudanças ao longo do tempo nos anos em que se desenvolveu e que se tornou um imperativo global na agenda da ONU. Sua concepção pode ser tanto antagônica à realização do direito à saúde, como apresentar algumas potencialidades, se observados alguns requisitos.

Palavras-chave: direito à saúde; cobertura universal de saúde, saúde global. 


\begin{abstract}
Universal health coverage was elevated to a highly prestigious position in the global health debate at the beginning of the 21st century, being indicated as the main goal in the sector. It starts with the discussion on expanding access, health-financing structires and is born closely related to the concepts of financial protection and equity, however, it starts to be mentioned in several international documents, until it becomes the main specific health-related goal, within the United Nations Sustainable Development Agenda, in addition to being the main reference for several other documents on health policy and health systems, especially within international organizations with an important influence on national policies, such as WHO, its regional offices, the World Bank and even civil society organizations. This work proposes to articulate the concept of universal health coverage within the framework of the realization of the right to health, in other words, we are aimed at understanding in what extent, it seeks to realize some of the aspects of the right to health and which. We take as a hypothesis that the realization of the right to health and universal coverage are not synonymous, but that they are often taken as such or as two ways to the same end, which would be to guarantee of health to all, which would result in two possible distortions. Restricting the realization of the Right to health to some of its elements or promoting a global health response to strengthen health systems that do not comply with the right to health. To this end, after proposing an identification of the elements of the Right to health, we conducted a documentary analysis of the resolutions and reports of the main WHO bodies. We conclude that universal coverage has changed over time in the years that it has developed and that it has become a global imperative on the UN agenda. Its conception can be both antagonistic to the realization of the right to health, as well as presenting some potentialities, if some requirements are observed.
\end{abstract}

Key-words: right to health; universal health coverage, global health 


\section{Lista de ilustrações}

Figura 1 - Representação esquemática dos principais elementos do direito à saúde

Figura 2 - Três dimensões da Cobertura universal de saúde no Relatório Mundial da Saúde de 2010

Figura 3 - Quadro de referência dos ODS a partir da Cobertura universal de Saúde extraído do relatório do Secretariado da OMS 
Lista de tabelas

Tabela 1 - Direito Sanitário e realização da cobertura universal segundo a OMS

Tabela 2 - Clausulas operativas da Resolução WHA58.33

Tabela 3 - Clausulas operativas da Resolução 64.9

Tabela 4 - Divisão do poder de voto no Banco Mundial no momento de sua fundação 


\section{Lista de abreviaturas e siglas}

Sistema Único de Saúde - SUS

Organização Mundial de Saúde - OMS

Assembleia Mundial de Saúde - AMS

Organização das Nações Unidas - ONU

Conselho Econômico e Social das Nações Unidas - ECOSOC

Organização Pan-Americana de Saúde - OPAS

Associação Internacional de Desenvolvimento - AID

Banco Internacional para Reconstrução e Desenvolvimento - BIRD

Corporação Financeira Internacional - CFI

Agência Multilateral de Garantia de Investimentos - AMGI

Centro Internacional para a Resolução de Controvérsias sobre Investimentos - ICSID

Fundo Monetário Internacional - FMI

Organização Mundial do Comércio - OMC

Acordo sobre Aspectos de Propriedade Intelectual relacionados ao Comércio - TRIPS

Atenção Primária à Saúde - APS

Organização para Cooperação e Desenvolvimento Econômico - OCDE

Objetivos de Desenvolvimento Sustentável - ODS

Objetivos de Desenvolvimento do Milênio - ODM 


\section{Sumário}

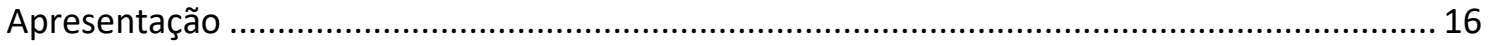

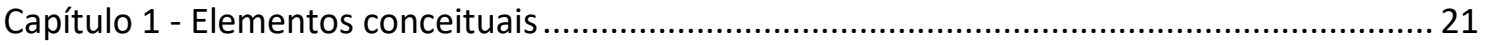

1.1 Apresentação do capítulo ...................................................................................... 21

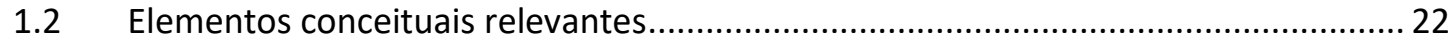

1.2.1 Sistemas de saúde e Serviços de saúde ...................................................................... 22

1.2.2 “Public Health”, Saúde Pública e Saúde Coletiva ......................................................... 24

1.2.3 Atenção Primária à Saúde e seus múltiplos sentidos, inclusive Atenção Básica ....... 27

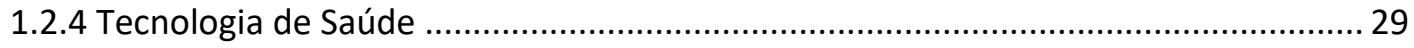

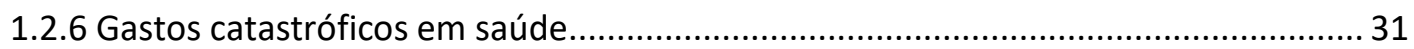

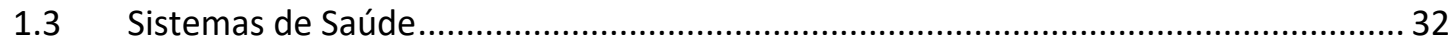

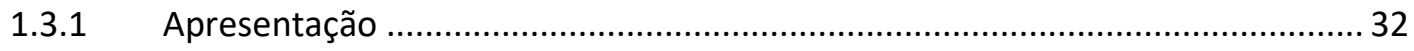

1.3.2 Elementos sobre a história dos sistemas de saúde........................................... 33

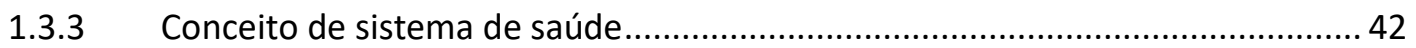

1.4 Saúde Global: elementos adicionais para compreensão das transformações ..............4 47

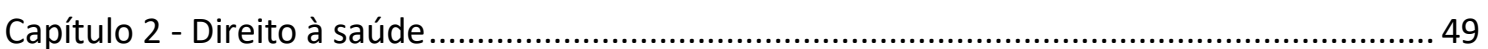

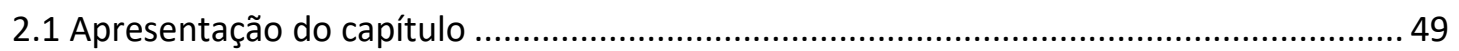

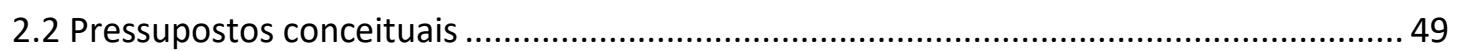

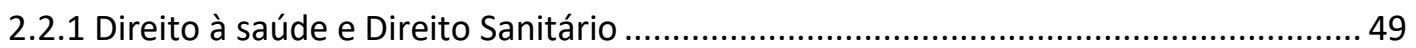

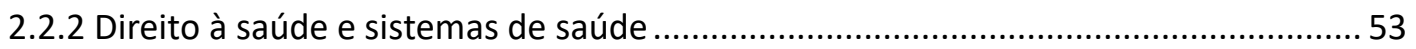

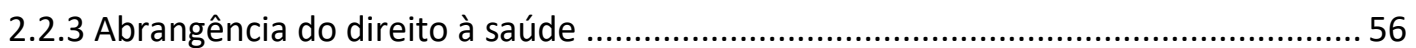

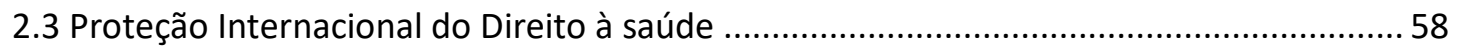

2.3.1 Declaração Universal dos Direitos Humanos ............................................................ 59

2.3.2 Pactos pelos Direitos Econômicos, Sociais e Culturais ...............................................60 60

2.3.3 Recomendação Geral n. 14, de 2000 - "O Direito ao mais alto nível alcançável de

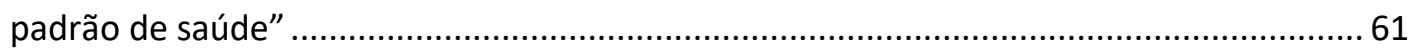

2.3.4 Constituição da OMS e Declaração de Alma-Ata ........................................................ 69

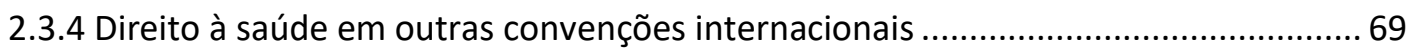

2.3.5 Direito à saúde, Acordo TRIPS e Declaração de Doha................................................. 70

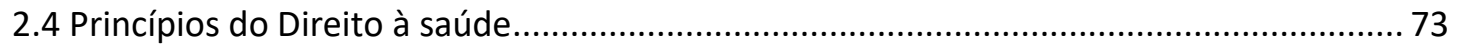

2.5 Direito à saúde e SUS: ilustração dos caminhos jurídicos para efetivação do Direito à

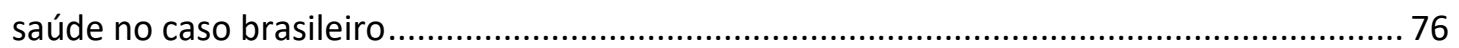

Capítulo 3 - Análise documental: Cobertura universal de saúde na OMS ................................ 49

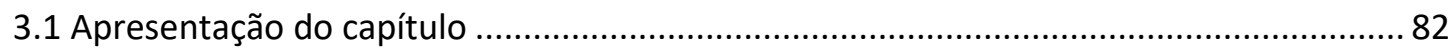

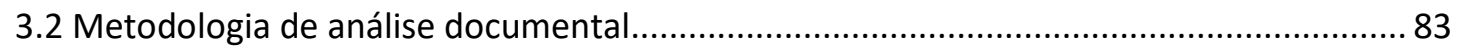

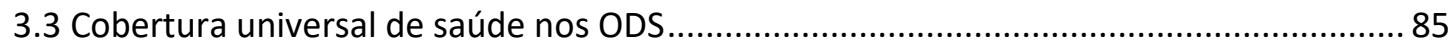




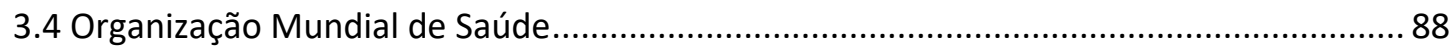

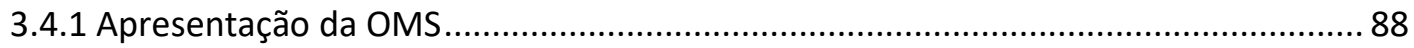

3.4.2 Relatório Mundial de Saúde de 2010 ………......................................................... 96

3.4.3 Análise documental da OMS - pré-Cobertura Universal de Saúde ......................... 102

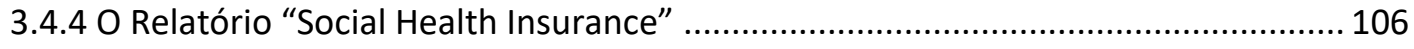

3.4.5 Análise documental da OMS - Cobertura Universal de Saúde ............................... 109

3.4.6 O Relatório Mundial da Saúde de 2008 e o Relatório da Comissão sobre

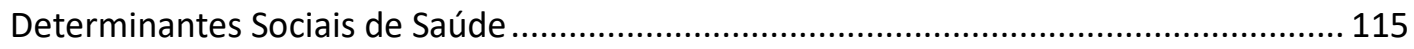

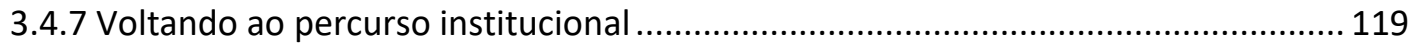

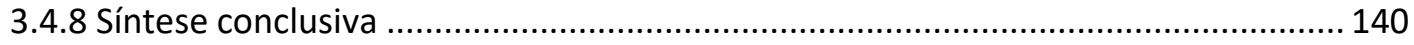

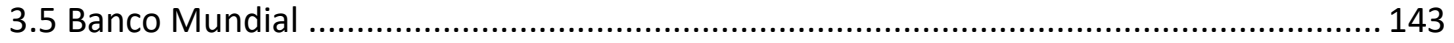

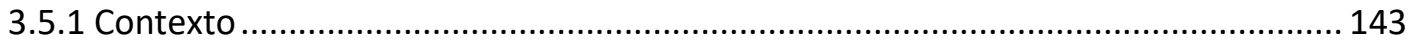

3.5.2 Banco Internacional de Reconstrução e Desenvolvimento ..................................... 143

3.5.3 Rastreando a Cobertura universal de saúde (2015, 2017 e 2019) - Banco Mundial,

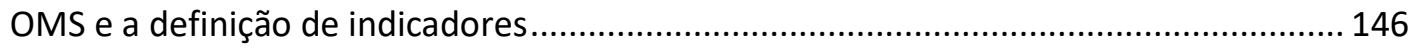

Capítulo 4: Cobertura universal de saúde e direito à saúde? .................................................. 153

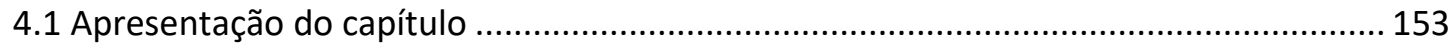

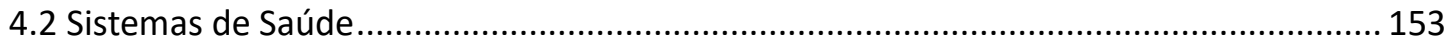

4.3 Afirmação do Direito à Saúde em relação aos Sistemas de Saúde .................................... 155

4.4 Afirmação e Definição do Direito à Saúde ....................................................................... 157

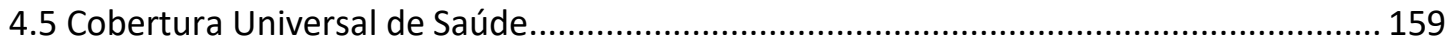

4.6 Cobertura Universal da Saúde e Direito à Saúde: Substituição ou Relação de Meio e Fim?

4.6.1 Cobertura universal de saúde vs. direito à saúde .................................................... 161

4.6.2 Cobertura universal de saúde e direito à saúde: relação de meio e fim ................... 168

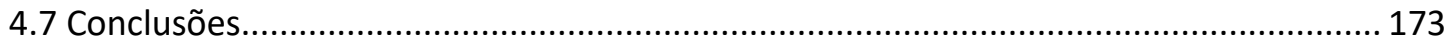

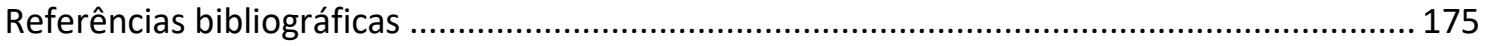




\section{Apresentação}

A Cobertura universal de saúde foi alçada a uma posição de alto prestígio no debate internacional sobre saúde no início do Século XXI, sendo indicada como o principal objetivo no setor e elemento central da Agenda da ONU para o Desenvolvimento Sustentável. Em 2012, a então Diretora-Geral da OMS, Margaret Chan, na abertura da $65^{\text {a }}$ Assembleia Mundial de Saúde declarou que "a cobertura universal de

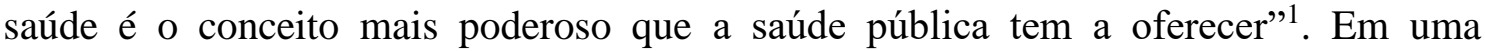
publicação editorial na prestigiosa publicação da área de saúde The lancet intitulada "Todos os caminhos levam para a Cobertura universal de saúde" ${ }^{2}$, em junho de 2017, o atual diretor Thedros Ghebreyesus, defende que:

\footnotetext{
"Todos os caminhos levam à Cobertura universal de saúde - e esta é a nossa principal prioridade na OMS. Para mim, a questão chave sobre a Cobertura universal de saúde é uma questão ética. Nós queremos que nossos concidadãos morram por que são pobres? Ou milhões de pessoas empobrecidas por gastos catastróficos em saúde por que não tem proteção contra o risco financeiro? A Cobertura universal de saúde é um direito humano." 3 (Grifos nosso)
}

Ela se inicia da discussão sobre ampliação do acesso, financiamento e sistemas de saúde e nasce intimamente ligada aos conceitos de proteção financeira e de equidade, no entanto, passa a ser mencionada em diversos documentos internacionais, até se tornar o principal objetivo específico associado à saúde na Agenda de Desenvolvimento Sustentável das Nações Unidas (Agenda 2030), além de principal referência para vários outros documentos sobre política sanitária e sistemas de saúde, especialmente no âmbito de organizações internacionais com importante influência sobre políticas nacionais, como a OMS, seus escritórios regionais, o Banco Mundial e até organizações da sociedade civil.

\footnotetext{
${ }^{1}$ Tradução livre de: "Universal health coverage is the single most powerful concept that public health has to offer" In: WORLD HEALTH ORGANIZATION. Address by Dr. Margaret Chan, Director-General to the Sixty-fifth World Health Assembly. 2012 Disponível em: <http://apps.who.int/gb/ebwha/pdf_files/WHA65/A65_3-en.pdf> Acesso em 23.jul.2019

2 GHEBREYESUS, Tedros Adhanom. All roads lead to universal health coverage. The Lancet Global Health, v. 5, n. 9, p. e839-e840, 2017.

3 Tradução livre de: "All roads lead to universal health coverage-and this is our top priority at WHO. For me, the key question of universal health coverage is an ethical one. Do we want our fellow citizens to die because they are poor? Or millions of families impoverished by catastrophic health expenditures because they lack financial risk protection? Universal health coverage is a human right."
} 
Este trabalho tem por objetivo analisar o conceito de cobertura universal de saúde no marco da efetivação do direito à saúde, em outras palavras, buscamos entender a relação entre os dois conceitos. Interessam-nos aspectos como, em que medida, ela busca realizar algum dos elementos do direito à saúde e quais ou mesmo qual é o significado para a efetivação do direito à saúde de a Cobertura universal tê-lo substituído em como imperativo máximo da proteção global à saúde.

Tomamos como hipótese que efetivação do direito à saúde e cobertura universal não são sinônimos, mas que muitas vezes são tomados como tal ou como duas vias para o mesmo fim, que seria garantir saúde a todas e a todos, o que resultaria em duas possíveis distorções: restrição da efetivação do direito à saúde a alguns de seus elementos ou promoção de uma resposta em saúde global para o fortalecimento dos sistemas de saúde que não condiz com o direito à saúde.

Para atender os objetivos e debater as duas hipóteses, buscamos propor uma identificação dos elementos do direito à saúde, ancorados na proteção internacional do direito à saúde por diversas convenções e documentos internacionais. Tomamos como referência para identificação de tais elementos tanto o referencial de afirmação histórica dos direitos humanos, inclusive econômicos e sociais, como o desenvolvimento de concepções políticas sobre a ideia de sistema de saúde, reconhecendo que este é o meio em que o direito à saúde se realiza, de modo que tão importante quanto a dimensão do direito em si é a dimensão que se tem da ideia de sistema.

Em seguida, realizamos análise documental das resoluções e relatórios das principais instâncias da OMS sobre o tema e de duas publicações da Organização publicadas em coautoria com o Banco Mundial. Ambas instituições são as principais promotoras do conceito de Cobertura universal de saúde e tem importante poder de influência, dentro do marco internacional, na formulação e implementação de políticas públicas e regulatórias a nível nacional.

O capítulo inicial tem a função de estabelecer um denominador comum para as discussões apresentadas nesta tese, sua função é conceituar alguns termos importantes ou apresentar o pano de fundo conceitual acerca de outros, fornecendo assim insumos para melhor compreensão do problema a ser descrito nas próximas etapas.

Passaremos assim pelos conceitos de sistemas de saúde, serviços de saúde (ou assistência à saúde), saúde pública e saúde coletiva, tecnologia de saúde, vigilância em saúde e gastos catastróficos em saúde. Esses são conceitos alheios ao universo do direito e típicos de outras áreas do conhecimento, como a saúde e a economia. 
Nosso objetivo é fornecer noções elementares sobre esses termos, que servirão de insumos indispensáveis para a compreensão da discussão seguinte. Neste capítulo também, duas seções são dedicadas ao aprofundamento sobre dois tópicos relevantes: sistemas de saúde e saúde global. Na discussão sobre sistemas de saúde, um documento central é o Relatório Mundial da Saúde de 2000, publicação referência da OMS, colocado em perspectiva e utilizado como ponto de partida da ideia de sistema de saúde, mas não como concepção final sobre o tema.

O capítulo seguinte tem por objetivo conceituar o direito à saúde. São apresentados aspectos de seu desenvolvimento histórico, seus elementos intrínsecos e a forma em que são utilizados no contexto de sua proteção internacional, com especial destaque para a Recomendação n. 14 do Conselho Econômico e Social das Nações Unidas, mas também a partir da discussão acadêmica sobre o tema. Pretendemos com esse capítulo esclarecer os caminhos que percorreremos para analisar o conceito ou mesmo os conceitos de cobertura universal de saúde.

Utilizamos para ilustrar este capítulo, a proteção jurídico-constitucional que o ordenamento brasileiro confere ao direito à saúde. A função principal dessa escolha é fornecer insumos para a discussão sobre a relação entre direito e cobertura universal. $\mathrm{O}$ Brasil reconhece a saúde enquanto direito e formulou em sua história recente um riquíssimo conjunto de elementos constitucionais associados a esse direito, sendo um dos melhores exemplos para ilustrar o conceito.

O quarto capítulo fornece o resultado da análise documental sobre Cobertura universal de saúde na OMS e em outras instâncias internacionais, especificamente a Assembleia Geral das Nações Unidas e declarações internacionais sobre o tema. Trata-se tanto de documentos formulado em linguagem jurídica, especialmente na forma de resoluções, quanto relatórios que subsidiam as discussões. Neste ponto, um documento em particular emerge com centralidade, o Relatório Mundial da Saúde de 2010, tendo uma seção específica dedicada a si. Buscamos também apresentar os elementos principais da governança da Organização.

Finalmente o capítulo final é dedicado à discussão das ideias apresentadas nos capítulos anteriores. Buscamos retomar de forma sintética, a relação entre sistemas de saúde, direito à saúde e cobertura universal, especialmente no intuito de articular estes dois.

A Cobertura universal de saúde pode ser compreendida, em determinados contextos, como um imperativo global que substitui, no plano político, a busca pelo 
direito à saúde. Nesse sentido, apresenta-se como um objetivo distinto e por vezes até antagônico à efetivação do direito. Não por implicar necessariamente em retrocessos ou violações, mas por reduzir o escopo da obrigação dos Estados em relação aos sistemas de saúde e até mesmo a ideia de saúde.

Em outro sentido, a Cobertura universal pode ser entendida como meio de realização do direito. Se assim for, há que se tomar o cuidado de que ela seja devidamente situada entre os vários elementos do direito à saúde e de que não viole o princípio do não retrocesso social ou leve à inclusão errônea de elementos da efetivação do direito sem relação com o problema que se propõe a resolver.

Nesta acepção, a estratégia global de cobertura deve ser adstrita ao que se propõe a enfrentar, isto é, o fim do risco financeiro, o que possivelmente não lhe conferiria a centralidade que tem, por exemplo, na Agenda 2030. 
comum que arranjos institucionais criados em um determinado contexto sociocultural não tenham a mesma performance em contextos distintos. Como conclusão, se a cobertura universal a nível local apontar para uma interação com o sistema securitário que priorize a função social do seguro, tendo como norte o direito à saúde, tais riscos podem ser minorizados e a segunda hipótese deste trabalho ganha força. Pelo contrário, a adoção por um modelo precipuamente mercadológico pode aproximá-la da primeira hipótese, de que a cobertura é uma substituição ao direito à saúde.

(iv) A articulação entre focalização de políticas e direito universal à saúde, sobo princípio da equidade

Vimos que o quadro de referência da Cobertura universal de saúde se vale de um conceito de equidade completamente distinto daquele que usamos como parâmetro ao conceituar o direito à saúde. Esta distinção pode ser compreendida também na chave da oposição entre sistemas universais de proteção social e políticas públicas focalizadas em grupos específicos.

Ainda que essa ideia indique oposição latente entre os dois principais conceitos estudados neste trabalho, chamamos atenção ao potencial de se combinar políticas públicas focalizadas com sistemas universais, utilizando-se inclusive conceitos transversais como determinantes sociais de saúde. O Programa Bolsa Família, que é uma iniciativa focalizada, é criado de forma articulada com o SUS, com o SUAS e com o sistema público de educação, indicando um exemplo de complementariedade. No mesmo sentido, a experiência indiana retratada acima serve de exemplo interessante.

A chave para o potencial da Cobertura universal de saúde estaria, nesse sentido, em considerá-la sempre dentro da referência mais ampla do direito à saúde e não tomar sua concepção de equidade como a única relevante dentro desse quadro. Nesse sentido, poderíamos formular ideias que se aproveitem da força da tendência global conferida à cobertura universal em favor da plena realização do direito à saúde.

\subsection{Conclusões}

O problema de se dar um valor tão grande à cobertura universal é que se pode promover o desmonte de sistemas já funcionais, com avanços significativos, institucionais e econômicos no sentido da universalização. O imperativo da Cobertura 
universal pode ser lido como a redução do dever dos Estados a um mínimo, de modo que mesmo o que se propunha como ponto de partida, torna-se o ponto de chegada.

Essa convicção, se lida em substituição ao direito à saúde enquanto verdadeiro imperativo, pode inclusive legitimar retrocessos em sistemas que já constituíram grandes avanços no sentido da universalização, o que contraria a ideia de não retrocesso social. Uma publicação que particularmente nos contempla nesse sentido foi publicada por Noronha ${ }^{206}$, com um título bastante significativo a respeito da substituição do direito pela cobertura como imperativo global.

Por outro lado, a cobertura universal, se lida dentro do contexto maior do direito à saúde é um conceito com algum potencial de contribuir para parte de sua realização. Seus principais problemas, nesse sentido, são a redução a um pacote básico de serviços, expresso especialmente nas publicações da OMS com o Banco Mundial, a redução do conceito de equidade a seus aspectos financeiros e a inclinação ao modelo securitário. Seus ganhos estariam em contextos específicos, de baixa penetração da proteção do direito à saúde, seja por limitações econômicas seja por concepções político-jurídicas.

\footnotetext{
${ }^{206}$ NORONHA, José Carvalho de. Cobertura universal de saúde: como misturar conceitos, confundir objetivos, abandonar princípios. Cad. Saúde Pública, Rio de Janeiro, v. 29, n. 5, p. 847-849, Maio 2013.
} 


\section{Referências bibliográficas}

ACOSTA, Angela, FALCÃO, Matheus Zuliane, AITH, Fernando Mussa Abujamra, et al. Judicialización del acceso a medicamentos en el contexto suramericano. Revista De Direito Sanitário, 2019, vol. 20, no 1, p. 32-62ALBERT, Michel. Capitalism against capitalism. John Wiley \& Sons Incorporated, 1993.

AITH, Fernando. Curso de direito sanitário: a proteção do direito à saúde no Brasil. 2007.

AITH, Fernando Mussa Abujamra. Teoria geral do direito sanitário brasileiro. 2006. Tese de Doutorado. Universidade de São Paulo.

AITH, Fernando. Políticas públicas de Estado e de governo: instrumentos de consolidação do Estado Democrático de Direito e de promoção e proteção dos direitos humanos. Políticas públicas: reflexões sobre o conceito jurídico. São Paulo: Saraiva, p. 217-245, 2006.

AGÊNCIA NACIONAL DE VIGIÂNCIA SANITÁRIA; ORGANIZAÇÃO MUNDIAL DE SAÚDE. Regulamento Sanitário Internacional: RSI - 2005. Versão em português aprovada pelo Congresso Nacional por meio do Decreto Legislativo 395/2009 publicado no DOU de 10/07/09, pág 11. Disponível em: < http://portal.anvisa.gov.br/documents/375992/4011173/Regulamento+Sanit\%C3\%A1rio +Internacional.pdf/42356bf1-8b68-424f-b043-ffe0da5fb7e5> p. 13. Acesso em 14.set.19.

ANJALI, S.; MAULIK, C. A realização do direito à saúde por meio da Cobertura Universal de Saúde. Revista de Direito Sanitário, v. 15, n. 2, p. 13-29, 6 jan. 2015.

AROUCA, Sergio. O dilema preventivista: contribuição para a compreensão e crítica da medicina preventiva. Unesp, 2003.

APPLEBY, John; DEVLIN, Nancy; PARKIN, David. NICE's cost effectiveness threshold. BMJ. 2007. Disponível em: < https://www.bmj.com/content/335/7616/358> Acesso em 24.out.2018.

BACHA, Stella Maris Cortez et OSÓRIO, Alda Maria do Nascimento. Fonoaudiologia e educação: uma revisão da prática histórica. Rev Cefac, 2004, vol. 6, no 2, p. 215-21.

BERAN, David, EWEN, Margaret, et LAING, Richard. Constraints and challenges in access to insulin: a global perspective. The lancet Diabetes \& endocrinology, 2016, vol. 4, no 3, p. 275-285.

BIEHL, João et PETRYNA, Adriana (ed.). When people come first: critical studies in global health. Princeton University Press, 2013. P. 102

BIEHL, João; SOCAL, Mariana P.; AMON, Joseph J. The judicialization of health and the quest for state accountability: Evidence from 1,262 lawsuits for access to medicines in Southern Brazil. Health and human rights, v. 18, n. 1, p. 209, 2016. 
BRASIL. Decreto Legislativo $n^{0}$ 395, de 9 de julho de 2009. Aprova o texto revisado do regulamento sanitário internacional, acordado na $58^{\mathrm{a}}$ Assembleia Geral da Organização Mundial de Saúde, em 23 de maio de 2005. Diário Oficial da União 2009

CONSELHO NACIONAL DE SAÚDE. Resolução n. 588, de 12 de julho de 2018. Disponível em: < http://conselho.saude.gov.br/resolucoes/2018/Reso588.pdf> Acesso em 16.jan.19

BRASIL; MINISTÉRIO DA SAÚDE. Portaria n. 2.436, de 21 de setembro de 2017. Aprova a Política Nacional de Atenção Básica, estabelecendo a revisão de diretrizes para a organização da Atenção Básica, no âmbito do Sistema Único de Saúde (SUS). Diário Oficial da União. Brasília. 2017

BRASIL. Decreto n. 4.377, de 13 de setembro de 2002. Promulga a Convenção sobre a Eliminação de Todas as Formas de Discriminação a Mulher, de 1979. 2002. Disponível em < http://www.planalto.gov.br/ccivil_03/decreto/2002/D4377.htm> Acesso em 15.ago.2019

BRASIL. Decreto n. 4.682, de 24 de janeiro de 1923. Crea, em cada uma das emprezas de estradas de ferro existentes no paiz, uma caixa de aposentadoria e pensões para os respectivos ernpregados. Disponível em : https://www2.camara.leg.br/legin/fed/decret/1920-1929/decreto-4682-24-janeiro-1923538815-publicacaooriginal-90368-pl.html > Acesso em 23.mai.2018

BRASIL. Decreto n. 65.810, de 8 de dezembro 1969. Promulga a Convenção Internacional sobre a Eliminação de todas as Formas de Discriminação Racial. Disponível em : <http://www.planalto.gov.br/ccivil_03/decreto/1950-1969/D65810.html> Acesso em 15.ago.2019

BRASIL. Decreto n. 99.710, de 21 de novembro de 1990. <http://www.planalto.gov.br/ccivil_03/decreto/1990-1994/D99710.htm> Acesso em 15.ago.2019

BRASIL. Portaria n. 2.436, de 21 de setembro de 2017. Aprova a Política Nacional de Atenção Básica, estabelecendo a revisão de diretrizes para a organização da Atenção Básica, no âmbito do Sistema Único de Saúde (SUS). Diário Oficial da União 2017

CLINTON, Hillary Rodham. Secretary of State. Remarks on Development in the 21st Century," Remarks at the Peterson Institute for International Economics, Washington, DC, 2010, vol. 6 .

COMPARATO, Fábio Konder. A afirmação histórica dos Direitos Humanos. São Paulo. Saraiva. 2008, $6^{\text {a }}$ ed. P. 58-9

CORREA, CARLOS. Public Health Perspective on Intellectual Property and Access to Medicines - a Compilation of Studies Prepared for WHO. South Centre, 2016.

CUBILLOS, Leonardo et al. Universal health coverage and litigation in Latin America. Journal of Health Organization and Management, v. 26, n. 3, p. 390-406, 2012 
DALLARI, Sueli Gandolfi. Uma nova disciplina: o direito sanitário. Revista de saúde pública, v. 22, p. 327-334, 1988.

DALLARI, Sueli Gandolfi. A democracia sanitária e o direito à saúde: uma estratégia para sua efetivação. JORNADA DE DIREITO À SAÚDE, I. Conselho Nacional de Justiça. Palestra. São Paulo, 2014

DALLARI, Sueli. O Sistema Único de Saúde. In: NUNES JR, Vidal Serrano (org.). Enciclopedia Jurídica da PUCSP. São Paulo. Pontificia Universidade Católica, 2017

DE ARAÚJO DOURADO, Daniel, DALLARI, Sueli Gandolfi, et ELIAS, Paulo Eduardo Mangeon. Federalismo sanitário brasileiro: perspectiva da regionalização do Sistema Único de Saúde. Revista de Direito Sanitário, 2012, vol. 12, no 3, p. 10-34.

DEERE, Carolyn. The implementation game: the TRIPS agreement and the global politics of intellectual property reform in developing countries. Oxford University Press, 2009.

ECOSOC. CESCR General Comment N. 14: The Right to the Highest Attainable Standard of Health. Disponível em: 〈https://www.refworld.org/pdfid/4538838d0.pdf> Acesso em 23.jul.2019.

FLEURY, S. et CARVALHO, Antônio Ivo de. Instituto Nacional de Assistência Médica da Previdência Social (INAMPS). FGV/CPDOC. Disponível em: http://www. fgv. br/cpdoc/acervo/dicionarios/verbete-tematico/instituto-nacional-de-assistenciamedicada-previdencia-social-inamps. Acesso, 2018, vol. 30.

FRIEDMAN, Eric, GOSTIN, Lawrence O., et BUSE, Kent. Advancing the right to health through global organizations: the potential role of a Framework Convention on Global Health. health and human rights, 2013, vol. 15, p. 71-86.

FILMER, Deon et PRITCHETT, Lant. The impact of public spending on health: does money matter?. Social science \& medicine, 1999, vol. 49, no 10, p. 1309-1323.

FLEURY, S. et CARVALHO, Antônio Ivo de. Instituto Nacional de Assistência Médica da Previdência Social (INAMPS). FGV/CPDOC. Disponível em: http://www. fgv. $\mathrm{br} / \mathrm{cpdoc/acervo/dicionarios/verbete-tematico/instituto-nacional-de-assistenciamedica-}$ da-previdencia-social-inamps. Acesso, 2018, vol. 30.

GIOVANELlA, Ligia, FEO, Oscar, FARIA, Mariana, et al. Sistemas de salud en Suramérica: desafíos para la universalidad la integralidad y la equidad. ISAGS, 2012.

GHEBREYESUS, Tedros Adhanom. All roads lead to universal health coverage. The Lancet Global Health, v. 5, n. 9, p. e839-e840, 2017.

GOSTIN, Lawrence O., BLAGOJEVIC, Aleksandra, BLAND, Simon, et al. Launching the Universal Health Coverage Legal Solutions Network. The Lancet, 2019. 
GOSTIN, Lawrence O., MONAHAN, John T., KALDOR, Jenny, et al. The legal determinants of health: harnessing the power of law for global health and sustainable development. The Lancet, 2019, vol. 393, no 10183, p. 1857-1910.

GOSTIN, Lawrence O. et al. The legal determinants of health: harnessing the power of law for global health and sustainable development. The Lancet, v. 393, n. 10183, p. 1857$1910,2019$.

GÖGH, Tibor. WTO Panel, Australia: Certain Measures Concerning Trademarks, Geographical Indications and Other Plain Packaging Requirements Applicable to Tobacco Products and Packaging, WT/DS435/R, WT/DS441/R, WT/DS458/R, WT/DS467/R, 28 June 2018. Legal Issues of Economic Integration, v. 46, n. 2, p. 182 196, 2019.

HOMEDES, Nuria. The disability-adjusted life year (DALY) definition, measurement and potential use. World Bank, 1996.

JÚNIOR, Nilson Martins Lopes. Direito Previdenciário: custeio e benefícios. Rideel, p.36, 2010

KEROUEDAN, Dominique. Géopolitique de la santé mondiale. Extraits de la leçon inaugurale prononcée le 14 février 2013. La lettre du Collège de France, 2013, no 36, p. 8.

LAUDE, Anne et al. Droit de la santé. Presses universitaires de France, 2007.

LEGGE, David. Future of WHO hangs in the balance. Bmj, v. 345, p. e6877, 2012.

MARKEL, Howard. Worldly approaches to global health: 1851 to the present. Public Health, v. 128, n. 2, p. 124-128, 2014.

MUSGROVE, Philp. Health insurance: the influence of the Beveridge Report. Bulletin of the World Health Organization, v. 78, p. 845-846, 2000NORONHA, José Carvalho de. Cobertura universal de saúde: como misturar conceitos, confundir objetivos, abandonar princípios. Cad. Saúde Pública, Rio de Janeiro , v. 29, n. 5, p. 847849, Maio $2013 \quad$ Disponível em: $<$ http://www.scielo.br/scielo.php?script=sci_arttext\&pid=S0102-

311X2013000500003\&lng=en\&nrm=iso>. Acesso em 20.ago.2018 http://dx.doi.org/10.1590/S0102-311X2013000500003.

MIRANDA, Pedro Henrique Marques Villardi. Da saúde internacional à saúde global: trajetórias históricas e geográficas do conceito de medicamentos essenciais. 2018.

OCKÉ-REIS, Carlos Octávio. SUS: o desafio de ser único. SciELO-Editora FIOCRUZ, 2012.

PAIM, JS. Nova Saúde Pública ou Saúde Coletiva? In: Desafios para a saúde coletiva no século XXI[online]. Salvador: EDUFBA, 2006, pp. 139-153. ISBN 978-85-2321177-6. Disponível em SciELO Books <http://books.scielo.org> 
PINTO, Élida G.; FLEURY, Sonia. Custeio do direito à saúde: em busca da mesma proteção constitucional conquistada pelo direito à educação. Revista de Direito Sanitário, Brasil, v. 13, n. 1, p. 54-80, june 2012. ISSN 2316-9044.

PIOVESAN, Flávia. Democracia, Direitos Humanos e globalização econômica: desafios e perspectivas para a construção da cidadania no Brasil. 2005.

GREENFIELD, Geva; FOLEY, Kimberley; MAJEED, Azeem. Rethinking primary care's gatekeeper role. Bmj, v. 354, p. i4803, 2016.

ORGANIZAÇÃO MUNDIAL DA SAÚDE; UNICEF. Declaração de Alma-Ata: Conferência Internacional sobre Cuidados Primário de Saúde. Alma-Ata. URSS. 1979.

https://web.archive.org/web/20090419121612/http://www.opas.org.br/coletiva/uploadA rq/Alma-Ata.pdf> Acesso em 15.set.19.

RAJAGOPAL, Balakrishnan. International law from below: Development, social movements and third world resistance. Cambridge University Press, 2003.

RAMOS, André de Carvalho. Processo internacional de direitos humanos. Editora Saraiva, 2017.p.88

ROEMER, Milton I. Social insurance for health service. Scandinavian journal of social medicine, v. 25, n. 2, p. 65-66, 1997.

ROSANVALLON, Pierre. La nouvelle question sociale. Repenser l'État-providence. Le seuil, 2015.

SCHILLING, Flávia. Um olhar sobre a violência da perspectiva dos direitos humanos: a questão da vítima. Revista IMESC, 2000, no 2, p. 59-65.

STARFIELD, Barbara. Primary care: balancing health needs, services, and technology. Oxford University Press, EUA, 1998.

SAVEDOFF, William D. Kenneth Arrow and the birth of health economics. Bulletin of the World Health Organization, v. 82, p. 139-140, 2004.

SENGUPTA, Amit. Promote the Health of All the People of the World: The Fortieth Newsletter (2018). 2018. $\quad$ Disponível em $<$ https://www.thetricontinental.org/newsletterissue/promote-the-health-of-all-thepeople-of-the-world-the-fortieth-newsletter-2018/> Acesso em 14.nov.2019.

TAYLOR, Alan John Percival. Bismarck - the man and the statesman. Penguin, p. 24, 1995

TUOHY, Carolyn Hughes, FLOOD, Colleen M., et STABILE, Mark. How does private finance affect public health care systems? Marshaling the evidence from OECD nations. Journal of health politics, policy and law, 2004, vol. 29, no 3, p. 359-396. 
UNITED NATIONS, Millennium. Millennium Declaration. UN General Assembly, report A/res/55/2, New York, 2000.

UNITED NATIONS GENERAL ASSEMBLY. Resolution adopted by the General Assembly on 25 September 2015:70/1. Transforming our world: the 2030 Agenda for Sustainable Development. Disponível em: <https://undocs.org/A/RES/70/1> Acesso em 24.jun.2018

UNITED NATIONS GENERAL ASSEMBLY. Resolution adopted by the General Assembly on 6 July 2017:71/313. Work of the Statistical Commision pertaining to the 2030 Agenda for Sustainable Development. Disponível em: $<$ https://undocs.org/A/RES/71/313> Acesso em 24.jun.2018

VENTURA D. Direito e Saúde Global: O caso da pandemia de gripe A (H1N1) São Paulo: Dobra Editorial; 2013.

VENTURA, Deisy; PEREZ, Fernanda Aguilar. Crise e reforma da organização mundial da saúde. Lua Nova, n. 92, p. 45-77, 2014.

WENDT, Claus. Mapping European healthcare systems: a comparative analysis of financing, service provision and access to healthcare. Journal of European Social Policy, 2009, vol. 19, no 5, p. 432-445.

WHO COMMISSION ON SOCIAL DETERMINANTS OF HEALTH et WORLD HEALTH ORGANIZATION. Closing the gap in a generation: health equity through action on the social determinants of health: Commission on Social Determinants of Health final report. World Health Organization, 2008

WORLD HEALTH ORGANIZATION, et al. Anchoring universal health coverage in the right to health: what difference would it make? Policy brief. 2015.

Year 2000. Genebra. $1981 . \quad$ Disponível em:
https://apps.who.int/iris/bitstream/handle/10665/38893/9241800038.pdf?sequence=1> Acesso em 11.out.2019

Poliomyelitis: Report by the Secretariat. Seventieth World Health Assembly. Provisional agenda item 12.3. Genebra. 2017.

. The world health report 2000: health systems: improving performance. World Health Organization, 2000.

et al. The world health report 2008: primary

health care now more than ever. 2008.

et al. WORLD HEALH REPORT (The):

Health Systems Financing: the path to universal Coverage. World Health Organization, 2010. 
WHA55.17 Dengue fever and dengue

haemorrhagic fever prevention and control. Fifty-fifth World Health Assembly. Genebra, 2002. Disponível em $<$ http://apps.who.int/gb/archive/pdf_files/WHA55/fwha5517.pdf $>$. Acesso em 27.mar. 2019.

WHA54.13 Strengthening health systems in developing countries. The Fifty-fourth World Health Assembly. Genebra, 2001. Disponível em <http://apps.who.int/gb/ebwha/pdf_files/WHA58-REC3/A58_REC3en.pdf\#page=189> . Acesso em 27.mar. 2019.

WHA60.29 Health Technologies. Sixtieth World Health Assembly. Genebra, 2007. Disponível em: < https://www.who.int/healthsystems/WHA60_29.pdf?ua=1> Acesso em 27.mar.19.

WHA61.14 Prevention and control of noncommunicable diseases: implementation of the global strategy. The Sixty-first World Health Assembly. Genebra, 2008. Disponível em $<$ https://www.who.int/ncds/governance/2008-resolution-which-endorsed-

GAP.pdf?ua=1 >. Acesso em 27.mar. 2019.

WHA64.8 Strengthening national policy dialogue to build more robust health policies, strategies and plans. Sixty-fourth World Health Assembly. Genebra, 2011. Disponível em <https://apps.who.int/gb/ebwha/pdf_files/WHA64-REC1/A64_REC1-en.pdf>. Acesso em 27.mar. 2019.

WHA64.9 Sustainable health financing structures and universal coverage. Sixty-fourth World Health Assembly. Genebra, 2011. Disponível em <https://apps.who.int/gb/ebwha/pdf_files/WHA64REC1/A64_REC1-en.pdf $>$. Acesso em 27.mar. 2019.

- WHA62.12 Primary health care, including health system strengthening. Sixty-second World Health Assembly. Genebra, 2009. Disponível em < https://www.who.int/hrh/resources/A62_12_EN.pdf $>$. Acesso em 27.mar. 2019.

WHA67.23 Health intervention and technology assessment in support of universal health coverage. Sixty-seventh World Health Assembly. Genebra, 2014. Disponível em < http://apps.who.int/gb/ebwha/pdf_files/WHA67/A67_R23-en.pdf>. Acesso em 27.mar. 2019.

. WHA67.24 Follow-up of the Recife Political Declaration on Human Resources for Health: renewed commitments towards universal health coverage. Sixty-seventh World Health Assembly. Genebra, 2014. 
Disponível em $<\quad$ http://apps.who.int/gb/ebwha/pdf_files/WHA67/A67_R24en.pdf?ua=1\&ua=1>. Acesso em 27.mar. 2019.

- WHA67.14 Health in the post-2015 development agenda. Sixty-seventh World Health Assembly. Genebra, 2014. Disponível em < http://apps.who.int/gb/ebwha/pdf_files/WHA67/A67_R14-en.pdf>. Acesso em 27.mar. 2019.

- WHA67.1 Global strategy and targets for tuberculosis prevention, care and control after 2015. Sixty-seventh World Health Assembly. $\quad$ Genebra, 2014. Disponível em https://apps.who.int/iris/bitstream/handle/10665/162760/A67_R1en.pdf?sequence $=1 \&$ is Allowed $=\mathrm{y}>$. Acesso em 27.mar. 2019.

- WHA67.12 Contributing to social and economic development: sustainable action across sectors to improve health and health equity. Sixty-seventh World Health Assembly. Genebra, 2014. Disponível em $<$ https://apps.who.int/iris/bitstream/handle/10665/162850/A67_R12-

en.pdf?sequence $=1 \&$ is Allowed $=\mathrm{y}>$. Acesso em 27.mar. 2019.

. WHA67.19 Strengthening of palliative care as a component of comprehensive care throughout the life course. Sixty-seventh World Health Assembly. Genebra, 2014. Disponível em < http://apps.who.int/medicinedocs/documents/s21454en/s21454en.pdf $>$. Acesso em 27.mar. 2019.

. WHA67.20 Regulatory system strengthening for medical products. Sixty-seventh World Health Assembly. Genebra, 2014. Disponível em $\langle$ https://apps.who.int/medicinedocs/documents/s21456en/s21456en.pdf $>$. Acesso em 27.mar. 2019.

Sixty-seventh World Health Assembly. Genebra, 2014. Disponível em $\langle$ http://apps.who.int/medicinedocs/documents/s21453en/s21453en.pdf $>$. Acesso em 27.mar. 2019.

WHA68.15 Strengthening emergency and essential surgical care and anaesthesia as a component of universal health coverage. Sixty-eighth World Health Assembly. Genebra, 2015. Disponível em <https://apps.who.int/gb/ebwha/pdf_files/WHA68/A68_R15-en.pdf>. Acesso em 27.mar. 2019.

- WHA68 Global vaccine action plan. Sixtyeighth World Health Assembly. Genebra, 2015. Disponível em < 
https://apps.who.int/iris/bitstream/handle/10665/253235/A68_R6-

en.pdf?sequence $=1 \&$ is Allowed=y $>$. Acesso em 27.mar. 2019.

- WHA68.20 Global burden of epilepsy and

the need for coordinated action at the country level to address its health, social and public knowledge implications. Sixty-eighth World Health Assembly. Genebra, 2015. Disponível em < https://apps.who.int/iris/bitstream/handle/10665/253249/A68_R20en.pdf?sequence $=1 \&$ is Allowed=y $>$. Acesso em 27.mar. 2019.

- WHA69.1 Strengthening essential public health functions in support of the achievement of universal health coverage. Sixtyninth World Health Assembly. Genebra, 2016. Disponível em < http://apps.who.int/gb/ebwha/pdf_files/WHA69/A69_R1-en.pdf>. Acesso em 27.mar. 2019.

- WHA69.11 Health in the 2030 Agenda for Sustainable Development. Sixty-ninth World Health Assembly. Genebra, 2016. Disponível em < http://apps.who.int/gb/ebwha/pdf_files/WHA69/A69_R11-en.pdf>. Acesso em 27.mar. 2019.

- WHA69.19 Global strategy on human resources for health: workforce 2030. Sixty-ninth World Health Assembly. Genebra, 2016. Disponível em < http://apps.who.int/gb/ebwha/pdf_files/WHA69/A69_R19en.pdf>. Acesso em 27.mar. 2019.

Tracking universal health coverage: first global monitoring report. World Health Organization, 2015.

\section{INTERNATIONAL BANK FOR RECONSTRUCTION AND DEVELOPMENT/THE WORLD BANK. Tracking universal health coverage: 2017 global monitoring report. World Health Organization and International Bank for Reconstruction and Development/The World Bank, 2017}

$\mathrm{XU}, \mathrm{Ke}$ et al. Household catastrophic health expenditure: a multicountry analysis. The lancet, v. 362, n. 9378, p. 111-117, 2003. 